\title{
TEORIA DO DÉFICIT DE AUTOCUIDADO: ANÁLISE DA SUA IMPORTÂNCIA E APLICABILIDADE NA PRÁTICA DE ENFERMAGEMA
}

\author{
Self-care deficit theory: analysis of importance and applicability in the nursing practice
}

Teoría del déficit del cuidado propio: análisis de su importancia y aplicabilidad en la práctica de la enfermería

\begin{abstract}
RESUMO
Objetivo: submeter a teoria do déficit de autocuidado de Orem a uma reflexão crítica. Metodologia: estudo teórico sobre os aspectos Importância e Aplicabilidade contidos no Modelo de Análise Crítica de Chinn e Kramer para análise crítica da teoria do déficit de autocuidado. Desenvolvido no período de outubro a dezembro de 2008. Resultados: o posicionamento da teoria do déficit de autocuidado está essencialmente relacionado à filosofia da enfermagem e demonstra potencial para influenciar ações de enfermagem, em especial relativas à educação para o autocuidado em pacientes portadores de cardiopatia isquêmica. Conclusões: a enfermagem, mediante a teoria do déficit de autocuidado, pode oferecer condições mais saudáveis e de maior autonomia ao indivíduo portador de cardiopatia isquêmica.
\end{abstract}

Palavras-chave: Enfermagem. Teoria de Enfermagem. Autocuidado. Isquemia Miocárdica.

\begin{abstract}
Purpose: was referring the Self-care deficit theory to a critical analysis. Methodology: a theoretical-reflection study about the aspects of Importance and Aplicability of Chinn and Kramer Critical Analysis Model about the Self-care deficit theory. It was developed between October and December 2008. Results: the Self-care deficit theory position is essentially related to the nursing philosophy and demonstrates potential to influence nursing actions, especially on education for the selfcare in patients with ischemic heart disease. Conclusions: the nursing upon the Self-care deficit theory can offer a more healthy conditions and autonomy to the individual with ischemic heart disease.
\end{abstract}

Keywords: Nursing. Nursing Theory. Self Care. Myocardial Ischemia.

\section{Resumen}

Objetivo: analizar la teoría del Déficit del autocuidado de Orem a un análisis crítico. Metodología: estudio teórico sobre los aspectos de la Importancia y la Aplicabilidad del modelo de análisis crítico de Chinn y Kramer sobre la teoría del Déficit del cuidado propio. Desarrollado en el período de octubre a diciembre de 2008. Resultados: El posicionamiento de la teoría del Déficit del cuidado propio es esencialmente relacionadas con la filosofía de la enfermería, y muestra el potencial para influir en las acciones de enfermería, en particular sobre la educación para la evaluación del propio paciente con cardiopatía isquémica. Conclusiones: la enfermería, en la teoría del Déficit del cuidado propio puede proporcionar una imagen más saludable y más autonomía a la persona con la enfermedad isquémica del corazón.

Palabras clave: Enfermería. Teoría de Enfermería. Autocuidado. Isquemia Miocárdica

\footnotetext{
${ }^{1}$ Enfermeira. Mestre em Enfermagem. Discente de Doutorado do Programa de Pós-Graduação em Enfermagem da Universidade Federal do Ceará- Fortaleza-CEBrasil. E-mail: allynefortes@yahoo.com.br,'2Enfermeiro. Doutor em Enfermagem. Professor Adjunto IV do Departamento de Enfermagem da Universidade Federal do Ceará- Fortaleza.-CE- Brasil. E-mail: marcos@ufc.br, ${ }^{3}$ Enfermeira. Doutora em Enfermagem. Professora Associada II do Departamento de Enfermagem Universidade Federal do Ceará- Fortaleza-CE-Brasil. E-mail: thelma@ufc.br
} 


\section{INTRODUÇÃO}

A teoria científica é definida como o conjunto de proposições sistematizadas, verificadas ou não para explicar determinado fenômeno, o que a torna o reflexo da realidade ou uma forma de representá-la. Ela parte da organização de hipóteses e leis dispersas, captadas com acuidade de percepção e síntese, em um corpo convergente e interpretativo. ${ }^{1}$

$\mathrm{Na}$ enfermagem, a teoria pode ser vista como resultado da percepção da realidade, da inter-relação de seus componentes, da formulação e da intercessão dos conceitos de ser humano, ambiente, saúde e cuidado de enfermagem. Teoria de enfermagem é então definida como uma conceitualização de algum aspecto da realidade de enfermagem cujo objetivo é descrever fenômenos, explicar as relações entre estes e predizer consequências ou prescrever o cuidado de enfermagem. ${ }^{2}$

Por conter elementos considerados fundamentais para a assistência, uma das teorias mais citadas na enfermagem brasileira é a denominada Déficit de Autocuidado, de autoria de Dorothea Orem, a qual faz parte do modelo de enfermagem proposto pela mesma autora. ${ }^{3}$

Com referência notadamente à teoria do déficit de autocuidado, como mostra a literatura, ela oferece subsídios ao cuidado por estar essencialmente apoiada na premissa segundo a qual todos possuem potencial, em diferentes graus, para cuidar de si mesmo e dos que estão sob sua responsabilidade. ${ }^{4,5}$ Entre os diversos contextos de sua aplicação, destaca-se o âmbito das doenças cardiovasculares, em virtude da possibilidade de comprometer a capacidade de autocuidado.

A incapacidade para desenvolver o autocuidado pode advir de fatores extrínsecos ou intrínsecos. Entre os primeiros, incluem-se a doença ou a morte de algum parente. Já fatores intrínsecos, como idade, por exemplo, afetam tanto a habilidade da pessoa se engajar no autocuidado, como o tipo e a quantidade de autocuidado requerido. ${ }^{5}$

Diante disso, surgiu o interesse em analisar a teoria do déficit de autocuidado, sobretudo no concernente à sua potencialidade para influenciar ações de enfermagem, em especial na população com o quadro clínico ora citado. Para complementar essa ideia, a crítica da teoria é definida como o exame crítico ou estimativo de uma coisa ou situação com o intuito de determinar sua natureza e limitações ou sua conformidade aos padrões. Nesse caso, as respostas respectivas a esse questionamento devem estar contidas na própria teoria., ${ }^{7}$

Portanto, o objetivo do presente estudo constituiu-se em submeter a teoria do déficit de autocuidado proposta por Orem a uma análise crítica. ${ }^{6}$

\section{Panorâmica sobre 0 modelo do autocuidado de Orem}

0 modelo do autocuidado proposto por Orem foi desenvolvido na década de 1950, baseado na premissa de que os indivíduos podem cuidar de si próprios. Contidas nele e entre si apresentam-se três teorias: a teoria dos sistemas de enfermagem; a teoria do déficit de autocuidado e a teoria do autocuidado. $^{8}$

Em linhas gerais, a teoria dos sistemas de enfermagem engloba a teoria do déficit de autocuidado e esta, por sua vez, contém a teoria do autocuidado. Quando uma exigência por cuidado de enfermagem é ativada, um sistema de enfermagem é produzido. Por isso, sistema de enfermagem é o conjunto de ações e interações dos enfermeiros e dos pacientes, classificado em totalmente compensatório, parcialmente compensatório e de apoio educativo. ${ }^{9}$

Como o conceito de déficit de autocuidado refere-se à relação entre o autocuidado e a exigência de autocuidado, está contido na teoria dos sistemas de enfermagem. Ele representa a necessidade de autocuidado, a qual, quando reconhecida, ativa um sistema de enfermagem. Deste modo, para a enfermagem ser legítima, o déficit de autocuidado precisa existir. ${ }^{6}$

Embutido no conceito apresentado, o autocuidado surge como o cuidado pessoal requerido pelos indivíduos cotidianamente para regular o próprio funcionamento e desenvolvimento. ${ }^{10} \mathrm{E}$ é justamente no comprometimento de alguns dos requisitos para o autocuidado que figura o déficit de autocuidado, explicitado adiante na apresentação da teoria do déficit de autocuidado, o cerne deste estudo.

\section{Teoria do déficit de autocuidado - o foco do estudo}

Esta teoria foi descrita pela primeira vez na segunda edição do modelo, pois na versão anterior fez-se referência apenas às dimensões do autocuidado. ${ }^{11}$ Naquele momento, o déficit de autocuidado não configurava uma teoria.

A teoria de enfermagem do déficit de autocuidado é 0 núcleo central da teoria geral de Orem, descrita, primeiro, em termos de suas funções, como uma teoria geral de enfermagem. Ela determina quando a enfermagem é necessária: sempre que um adulto ou pai/responsável (no caso de um dependente) é inábil ou se encontra limitado no suprimento de autocuidado eficaz continuado. ${ }^{9}$

Assim, as oito funções da teoria de enfermagem do déficit de autocuidado são: definir que termos referentes ao ser humano são mais adequados à enfermagem; destacar o enfoque de enfermagem mais adequado; estabelecer uma linguagem própria; definir limites para orientar 0 pensamento, a prática, a investigação e a educação; reduzir a carga cognitiva, proporcionando subsídios à razão para receber informações e permitir às pessoas categorizar conceitos de forma a relacionar insights sobre características de situações concretas de enfermagem; permitir inferências sobre as articulações da enfermagem com outros domínios da atividade humana; gerar nos estudantes e nos enfermeiros um estilo de pensamento e comunicação padronizados; e inserir os enfermeiros no âmbito acadêmico. 


\section{METOLOGIA}

\section{Natureza e período de estudo}

Trata-se de um estudo de natureza teórica desenvolvido nos meses de outubro, novembro e dezembro de 2008 mediante a aplicação do Modelo de Análise Crítica da Teoria proposto por Chinn e Kramer para a análise crítica da teoria do déficit de autocuidado. ${ }^{7,5}$ Dentre os componentes do modelo, elegeram-se para a análise os componentes Importância e Aplicabilidade tendo em vista a exigência de uma teoria estar ligada à sua significância clínica, valor e aplicação na prática profissional.

Para a execução do estudo, primeiramente procedeu-se à leitura da teoria do déficit de autocuidado de Orem, em sua quinta edição, seguida da análise crítica da teoria com ênfase nos componentes citados anteriormente. ${ }^{6}$

\section{Referencial metodológico: Modelo de Análise Crítica da Teoria}

As análises de teorias sob o Modelo de Análise Crítica da Teoria proposto por Chinn e Kramer abrangem a descrição da teoria e a análise crítica teórica. ${ }^{7}$ Conforme a situação, o modelo de análise selecionado pode ser empregado em partes ou no todo, propiciando escolher enfocar qualquer um dos componentes de análise ou descrição. Segundo esse modelo menciona, a análise de uma teoria pode ser realizada de duas formas: por meio do guia de descrição ou pelo processo de análise crítica sobre a teoria. ${ }^{7}$ No presente trabalho, optou-se pela análise crítica formada pelos componentes: Clareza, Simplicidade, Generalidade, Acessibilidade, Importância e Aplicabilidade, sendo os dois últimos adotados no estudo. A análise crítica difere da simples descrição, pois nesse âmbito é possível qualificar a teoria e verificar se ela, de fato, corresponde ao propósito de quem vai aplicá-la, seja na pesquisa, na prática ou na educação. ${ }^{6}$

Estabeleceu-se, então, a seguinte questão central para análise: "A teoria é aplicável no sentido de criar uma realidade importante para a enfermagem?" Para responder à questão central, o modelo de análise adotado apresenta quatro perguntas, das quais duas foram selecionadas e empregadas ao tema do estudo: A teoria do déficit do autocuidado tem potencial para influenciar ações de enfermagem?; 0 posicionamento da teoria do déficit do autocuidado sobre pessoas, enfermagem e ambiente está consistentemente relacionado com a filosofia da enfermagem? As respostas para as questões escolhidas dentro do componente considerado devem ser respondidas pela própria teoria.

\section{ANÁLISE E INTERPRETAÇÃO DE RESULTADOS}

\section{Análise crítica da teoria do déficit de autocuidado de acordo com o Modelo de Análise Crítica da Teoria descrito por Chinn e Kramer?.}

A análise crítica da teoria do déficit de autocuidado foi desenvolvida com ênfase nas duas perguntas escolhidas e está apresentada nos subitens a seguir. ${ }^{6}$

A teoria do déficit de autocuidado tem potencial para influenciar ações de enfermagem? Se sim, para qual finalidade? Esse fim é desejável?

Para a teoria do déficit de autocuidado, a causa das ações de enfermagem ao indivíduo está associada à intenção de torná-lo completamente ou parcialmente capaz de saber regular cuidados para si ou para seus dependentes e conseguir empenhar-se na continuação do desempenho dessas medidas para controlar ou, de alguma forma, gerir fatores que interferem no funcionamento e desenvolvimento próprios ou de seus dependentes. ${ }^{10}$

Na primeira edição do modelo de Orem, ${ }^{10}$ os tipos de ações apontados pela teoria indicam: ajustar meios de atender às necessidades universais de autocuidado; estabelecer novas técnicas para o autocuidado; modificar a autoimagem; revisar a rotina de vida diária; desenvolver um novo estilo de vida compatível com os desvios da saúde; promover o enfrentamento dos efeitos do desvio da saúde ou da terapêutica médica. ${ }^{11}$

$\mathrm{Na}$ edição seguinte, a qual propõe de fato a teoria do déficit do autocuidado, as ações recomendadas pela teorista são delineadas em quatro atividades gerais: ajudar a iniciar e manter relacionamentos do paciente com indivíduos, famílias ou grupos até esse poder se desligar dos cuidados de enfermagem; determinar se e como os pacientes podem ser auxiliados pela enfermagem; responder aos questionamentos, anseios e necessidades do paciente por contato e assistência de enfermagem; prescrever, prover e regular ajuda direta na rotina de vida diária do paciente, nas necessidades social, educacional e de cuidado. ${ }^{12}$

Na edição adotada para análise, a teoria do déficit de autocuidado foi acrescida de alguns princípios que englobam e transpõem as ações gerais. Para a teorista, o enfermeiro deve lidar com os detalhes de cada caso sem jamais sobrepujar-se às conceituações universais nas quais as ações de enfermagem estão embasadas, ao considerar seu domínio, seus elementos e os tipos de resultados a serem alcançados. Isto inclui as normas e padrões da prática na qual se reúnem os conhecimentos necessários para a realização das ações e sua organização, sempre de acordo com os resultados esperados. ${ }^{6}$

Ao considerar a população foco deste estudo, segundo observado, esses indivíduos apresentam necessidades específicas quanto ao autocuidado. Um estudo sobre a prática do autocuidado essencial após a revascularização do miocárdio aponta o próprio déficit de autocuidado como um 
fator de risco expressivo para o desenvolvimento de doenças coronarianas. Nele é mencionada a responsabilidade do enfermeiro em fornecer as devidas orientações neste sentido. ${ }^{13}$

Em outra pesquisa, a teoria do déficit de autocuidado é considerada benéfica para direcionar as ações assistenciais do enfermeiro e responder às necessidades de autocuidado de pacientes com doenças cardiovasculares.?

Diversos estudos confirmam a importância do uso da teoria do déficit de autocuidado de Orem no referente aos pacientes portadores de alguma cardiopatia isquêmica com a finalidade de contribuir para individualização, humanização e qualificação das ações de enfermagem, além de promover o autoconhecimento, o autocontrole e a participação dos pacientes no próprio cuidado. ${ }^{5}$

Uma análise crítica acerca do uso da teoria do déficit de autocuidado de Orem como um meio para responder eficazmente às necessidades dos pacientes com alterações coronarianas, internados e sob cuidados do enfermeiro, afirma que embora complexa, tanto na linguagem como construção, a teoria fornece uma abordagem abrangente e holística para as ações voltadas a essas pessoas. ${ }^{13}$

Em suma, as ações sugeridas pela teoria do déficit de autocuidado na edição analisada podem se resumir às seguintes iniciativas: elaboração de métodos de auxílio, ações voltadas para agir ou fazer para outra pessoa, conduzir e orientar, oferecer apoio físico e psicológico, adequar o ambiente ao desenvolvimento pessoal e, por fim, ações focalizadas no ensino.

Neste prisma, a teoria do déficit de autocuidado pode ser entendida como um exemplo válido de descrição da enfermagem como uma ciência voltada para a prática e favorável à aplicação em pacientes portadores de cardiopatia isquêmica. Seu potencial para influenciar ações de enfermagem foi notoriamente percebido, em especial quanto às ações de educação para o autocuidado dessa clientela.

0 posicionamento da teoria do déficit de autocuidado sobre pessoas, enfermagem e ambiente está consistentemente relacionada com a filosofia da enfermagem?

Filosofia é uma forma disciplinada de investigação que distingue traços gerais de uma realidade na qual princípios e valores estão alicerçados. Historicamente, o desenvolvimento do conhecimento de enfermagem apoiou-se essencialmente em pressupostos da filosofia ao incluir teorias sobre a natureza da realidade, a natureza do conhecimento e meios para discernir realidade, princípios e valores. ${ }^{4}$ A pessoa, 0 ambiente, a saúde e a enfermagem foram propostos como fenômenos de enfermagem e, ao mesmo tempo, como os primeiros conceitos metaparadigmáticos de enfermagem e continuaram a ter utilidade na condição de principais conceitos de organização para a disciplina e para a profissão. ${ }^{2}$

De tal modo, a filosofia da enfermagem tem por função especificar as definições de conceitos do metaparadigma em cada um dos modelos conceituais da enfermagem. Pelo modelo de análise crítica de teorias adotado, discutiram-se então os conceitos metaparadigmáticos, pessoa, enfermagem e ambiente, sob a visão da teoria do déficit de autocuidado. ${ }^{6,8}$

\section{0 posicionamento da teoria do déficit de autocuidado sobre pessoas com cardiopatias isquêmicas}

Durante a $5^{\text {a }}$ Conferência Anual de Pós-Mestres em 1973, Orem divulgou cinco proposições sobre ser humano que embasariam as demais edições da teoria. ${ }^{12}$ Portanto, cinco premissas sobre autocuidado e ser humano serviram como princípios norteadores em todo o processo de conceitualização da teoria do déficit de autocuidado.

$1^{0}: 0$ ser humano exige contínua e deliberadamente insumo para si próprio e para seu ambiente, com o objetivo de permanecer vivo e em acordo com a função humana natural.

Quanto a isso, os indivíduos portadores de cardiopatia isquêmica apresentam comumente deficiência na oxigenação tissular, o que causa intolerância à atividade, comprometendo seu autocuidado em diferentes graus. ${ }^{14}$

$2^{0}$ : 0 ser humano tem o poder de agir de maneira autônoma, e o exerce sob a forma de cuidados de si próprio e de terceiros, caso haja a identificação de necessidades ou de insumos necessários.

Neste sentido, a cardiopatia isquêmica pode intervir negativamente na capacidade de autocuidado do indivíduo e, por conseguinte, em seu desempenho de papel familiar e social. ${ }^{14}$

$3^{0}: 0$ ser humano em processo de envelhecimento experimenta limitações para ações no cuidado de si mesmo e relacionadas à provisão de insumos de sobrevivência como à própria função regulatória do corpo.

Por isso, a aplicabilidade da teoria do déficit de autocuidado é ressaltada, visto que o envelhecimento da população em todo o mundo e determinadas doenças constituem hoje um problema social da maioria das comunidades, particularmente as enfermidades cardiovasculares, detentoras de liderança no acometimento em idosos. ${ }^{15}$

$4^{0}$ : 0 organismo humano é estimulado a descobrir, desenvolver meios e transmitir aos outros suas necessidades de insumos.

Assim, a escuta ativa do profissional ou mesmo de pessoas amigas torna-se fundamental ao auxiliar o paciente a se autodescobrir e comunicar suas necessidades. Isso pode fornecer uma sensação de alívio e bem-estar ao indivíduo em tratamento de cardiopatia isquêmica. ${ }^{6}$

$5^{0}$ : Grupos de seres humanos com relações estruturadas delegam tarefas e imputam as responsabilidades da prestação de cuidados aos membros do grupo que vivenciam alguma dificuldade de autocuidado.

Neste aspecto, as redes de apoio, tanto emocional quanto funcional, podem intervir no déficit de autocuidado desses pacientes mediante sentimentos de solidariedade, 
confiança, valorização e melhor adequação do indivíduo às situações difíceis, a exemplo dos problemas cardiovasculares. ${ }^{?}$ Pesquisas nessa área corroboram o valor do papel dos familiares como agentes colaboradores em face da possibilidade de influenciar diretamente no tratamento, nos comportamentos de autocuidado e na mudança do estilo de vida. ${ }^{13}$ Nota-se a intenção mais evidente da teoria em considerar, no enfoque do déficit de autocuidado, família ou grupos/comunidades como agentes de autocuidado.

\section{0 posicionamento da teoria do déficit de autocuidado sobre enfermagem}

Na primeira edição do seu modelo teórico, Orem definiu a enfermagem como um meio, um serviço para superar as limitações humanas. ${ }^{11}$ Na edição subsequente, a enfermagem é vista sob o enfoque do desenvolvimento da arte, que vai além das habilidades, conhecimentos e atitudes. ${ }^{12}$

A terceira edição sintetiza quatro pontos de vista sobre o conceito de enfermagem: o cuidado provido por enfermeiros a indivíduos em diversas situações e produzido de acordo com as leis que o regem; um conhecimento particular; um tipo de arte singular que qualifica o enfermeiro; e um campo de trabalho, vocação, ocupação. ${ }^{10}$

$\mathrm{Na}$ edição adotada para esta análise crítica, a enfermagem é referida mais consistentemente como um campo de instrução de conhecimentos com componentes teóricos e práticos, embora notadamente mais orientados para a prática em si.

Sobre a contribuição de Orem na assistência a pacientes com problemas cardíacos, segundo afirma determinado estudo, a enfermagem pode ser vista como promotora do autocuidado como autoconhecimento e autogoverno, constituindo uma área capaz de oferecer um diferencial na adaptação à nova condição de vida de tais clientes. ${ }^{14}$

Por conseguinte, como se percebe, o conceito de enfermagem na visão de Orem foi construído contínua e progressivamente no decorrer do tempo, do aprimoramento da teoria e da transformação da própria realidade que a envolve e atuou como fundamento para a composição dos demais conceitos por ela tratados.

0 posicionamento da teoria do déficit de autocuidado sobre ambiente para pessoas com cardiopatias isquêmicas

De forma discreta, o déficit de autocuidado reporta-se ao ambiente como âmbito social da saúde quando se refere à ideia de dependência social. Isso torna o cuidado de enfermagem um meio de promoção, recuperação e reabilitação da autonomia social do indivíduo e, consequentemente, de sua saúde. No âmbito do desenvolvimento de tecnologias, propõe as que permitam o controle de posição e movimento de pessoas nos seus ambientes físicos, a denotar sua preocupação quanto à influência da relação do ser humano com o ambiente sobre sua capacidade de autocuidado. ${ }^{9}$
Um estudo similar refere o ambiente no qual o indivíduo com problemas cardiovasculares está inserido, especialmente as influências socioculturais e o núcleo familiar, como fatores condicionantes que afetam tanto a habilidade da pessoa de se engajar no autocuidado como o tipo e a quantidade de autocuidado requerido. ${ }^{7}$

Como evidenciado, esse conceito corrobora a ideia preconizada na teoria, e, como integrantes de uma rede social de apoio, a família, grupos e comunidades são componentes importantes do ambiente do indivíduo portador de cardiopatia isquêmica. Por isso, a inclusão da família no tratamento hospitalar e no acompanhamento subsequente é decisiva no concernente à disposição para o autocuidado dessas pessoas.

De maneira geral, conforme compreendido, o posicionamento da teoria do déficit de autocuidado está essencialmente relacionado à filosofia da enfermagem. Esta concepção é corroborada por outro estudo ao destacar a contribuição potencial da aplicação dessa teoria para a definição de cuidados a pessoas com cardiopatias isquêmicas a partir de uma perspectiva prática e filosófica condizente com a própria filosofia da enfermagem. ${ }^{14}$

\section{CONCLUSÕES}

Com base na análise reflexiva realizada, pode-se concluir que a teoria do déficit de autocuidado proposta por Orem tem importância e é aplicável a indivíduos portadores de cardiopatia isquêmica, além de apresentar potencial para influenciar ações de enfermagem em outras situações nas quais 0 autocuidado esteja deficiente.

Sua importância fundamenta-se no fato de que contém conceitos, definições, propósitos e suposições baseados na prática e, por isso, possui valor para melhorar a prática ao ressaltar a afinidade do enfermeiro com a teoria, na relação de seu objetivo, recuperar o autocuidado, e o cerne da enfermagem, o cuidado. Assim, considera-se que toda ação de enfermagem que vise recuperar o autocuidado pleno do indivíduo poderá remontar a importância dos pressupostos, métodos e metas da teoria do déficit de autocuidado proposta por Orem.

0 cuidado propiciado a essa população deve oferecer assistência às necessidades físicas, apoio psicológico, além de incluir estratégias que ofereçam 0 autoconhecimento, 0 autocontrole e a participação ativa dessas pessoas no próprio cuidado. Neste sentido, o enfermeiro tem o papel de promover, manter e restaurar o conforto, sobretudo com relação à dor anginosa, mediante a manutenção da oxigenação, o controle medicamentoso, adequação do ambiente, diminuição dos fatores estressores e promoção do enfrentamento.

As principais ações de autocuidado a serem aplicadas a esses pacientes pela enfermagem podem centrar-se notadamente nas ações educativas, em especial 
sobre: a isquemia cardíaca, suas causas e consequências; os tratamentos aos quais serão submetidos, suas implicações imediatas e tardias; as mudanças necessárias no estilo de vida com vistas ao controle dos fatores de risco extrínsecos e intrínsecos, o reconhecimento de sinais e sintomas que evidenciem novas crises e as medidas necessárias a serem tomadas, as reais possibilidades e limitações de cada indivíduo após a alta.

De acordo com o previsto, a enfermagem, mediante o ensino do autocuidado, juntamente com o tratamento convencional, tem a possibilidade de propiciar condições mais saudáveis e de maior autonomia ao indivíduo portador de cardiopatia isquêmica. Consequentemente, será possível reduzir complicações e reincidências. Devidamente administradas, as ações de autocuidado da enfermagem podem não só promover alívio do sofrimento, mas principalmente preveni-lo. Isso certamente proporcionará melhores condições durante a permanência do paciente no ambiente hospitalar, além de evitar a necessidade de ser submetido a outras intervenções e a um período mais longo de internação.

Por fim, ao considerar a teoria do déficit de autocuidado proposta por Orem, sua importância e aplicabilidade no ensejo das cardiopatias isquêmicas, entende-se que o enfermeiro tem possibilidade de aplicá-la às outras situações em que 0 autocuidado do indivíduo esteja deficiente e, por conseguinte, demande algum cuidado de enfermagem

\section{REFERÊNCIAS}

1.Viegas W. Fundamentos lógicos da metodologia científica. Brasília(DF): Universidade de Brasília; 2007

2.Meleis Al. Theoretical nursing: development and progress. Philadelphia: Lippincont; 1997.

3. Costa LB, Costa AAS, Saraiva MRB, Barroso MGT. Appliation of conceptual structure in the nursing consultation to the family. Esc Anna Nery Rev Enferm 2007 set; 11(3): 515-19.

4.Caetano JA, Pagliuca LMF. Descriptive analysis of the nursing systems theory of orem before it's application concerning ocular self-exam teaching. Esc Anna Nery Rev Enferm 2003 abr; 7(1): 89-96.

5.Lima FET, Araujo TL. Prática do autocuidado essencial após a revascularização do miocárdio. Rev Gaucha Enferm 2007; 28(2): 22332.

6.Orem DE. Nursing: concepts of practice. St Louis: Mosby; 1995.

7.Cade NV. A teoria do déficit de autocuidado de Orem aplicada em hipertensas. Rev Latino-am Enfermagem 2001; 9(3): 43-50.

8.Chinn PL, Kramer MK. Theory and nursing: a systematic approach. Missouri: Mosby; 1995.
9.Orem DE. Modelo de Orem: conceptos de enfermeria en la practica. Barcelona: Masson-Salvat; 1993.

10.Orem DE. Nursing: concepts of practice. New York: McGraw-Hill; 1985.

11.Orem DE. Nursing: concepts of practice. New York: McGraw-Hill; 1971.

12.Orem DE. Nursing: concepts of practice. New York: McGrau-Hill; 1980.

13.Timmins F, Horan P. A critical analysis of the potential contribution of Orem's self-care deficit nursing theory to contemporary coronary care nursing practice. Eur J Cardiovasc Nurs 2007; 6(1): 32-9.

14.Lima LR, Pereira SVM, Chianca TCM. Diagnósticos de Enfermagem em pacientes pós-cateterismo cardíaco: contribuição de Orem. Rev Bras Enferm 2006; 59(3): 285-90.

15.Freire MRSM. The hypertense aged people and the self-care. [tese de doutorado]. João Pessoa (PB): Universidade Federal da Paraíba; 2000. 